\title{
ATP-dependent remodeling and acetylation as regulators of chromatin fluidity
}

\author{
Robert E. Kingston ${ }^{1}$ and Geeta J. Narlikar \\ Department of Molecular Biology, Massachusetts General Hospital, Boston, Massachusetts, 02114 USA; Department \\ of Genetics, Harvard Medical School, Boston, Massachusetts 02115 USA
}

It has become widely accepted that modification of nucleosome structure is an important regulatory mechanism. The hypothesis that the acetylation of histones is involved in regulation was first formed over thirty years ago by Allfrey and colleagues (Allfrey et al. 1964). Subsequent genetic studies suggested that complexes that utilize ATP hydrolysis to alter chromatin structure might also play a regulatory role. In the past 5 years, numerous ATP-dependent remodeling complexes, acetyltransferases, and acetyltransferase complexes have been isolated and characterized. With the identification of these complexes, it is now possible to examine how these complexes modulate gene expression, and how the action of these complexes can be coordinated.

The two major classes of chromatin modifying complexes that have been characterized differ in whether or not they use covalent modification to alter chromatin structure (recent reviews include Felsenfeld et al. 1996; Hartzog and Winston 1997; Tsukiyama and Wu 1997; Gregory and Horz 1998; Imhof and Wolffe 1998; Kadonaga 1998; Kuo and Allis 1998; Mizzen and Allis 1998; Pollard and Peterson 1998; Varga-Weisz and Becker 1998; Workman and Kingston 1998). The first class consists of histone acetyltransferase (HAT) and deacetylase complexes, which, respectively, add and remove acetyl groups from the amino termini of the four core histones; increased acetylation is usually (but not always) associated with activation of gene expression, whereas decreased acetylation is associated with repression of gene expression. The second class consists of ATP-dependent chromatin remodeling complexes, which alter chromatin structure by changing the location or conformation of the nucleosome. These structural changes are accomplished without covalent modification, and can be involved in either activation or repression. In addition to these two major classes of complexes, there are also other recently identified complexes such as FACT, DRIP/ARC, and SPT4/SPT5 that help the transcription machinery contend with nucleosome structure (Hartzog

${ }^{1}$ Corresponding author.

E-MAIL kingston@frodo.mgh.harvard.edu; FAX (617) 726-5949. et al. 1998; LeRoy et al. 1998; Orphanides et al. 1998; Wada et al. 1998; Naar et al. 1999; Rachez et al. 1999). The mode of action of these other complexes is not yet understood.

This review focuses on ATP-dependent remodeling complexes and how these complexes interact with acetylation complexes to regulate gene expression.

\section{Timing}

An important issue is the order in which ATP-dependent remodeling complexes and acetyltransferases function relative to each other and relative to transcription processes. Does a change in acetylation status of any single nucleosome precede or follow ATP-dependent remodeling? Which steps in the transcription process occur prior to these modifications, and which occur following these modifications?

Despite the obvious importance of these questions, there is currently very little concrete data on these issues. The most detailed information about the temporal ordering of these complexes on a promoter comes from recent studies of the Saccharomyces cerevisiae HO promoter. The activity of this promoter is regulated across the cell cycle. By synchronizing yeast and using chromatin immunoprecipitation studies to characterize when different proteins are bound to the promoter, two groups were able to order events on the HO promoter (Cosma et al. 1999; Krebs et al. 1999). Both the SWI/SNF remodeling complex (discussed in detail below) and the SAGA acetyltransferase complex bind to the $\mathrm{HO}$ promoter following transient binding by the SWI5 activator and before binding by the activator heterodimer SWI4/SWI6 (Cosma et al. 1999). Analysis of binding in swi/snf mutants showed that SWI/SNF is required for binding of the SAGA complex. Importantly, SWI/SNF is also required to observe increased acetylation of the promoter, which occurs roughly concurrently with SAGA binding (Krebs et al. 1999). Binding by both SWI/SNF and SAGA occurs prior to transcriptional activation.

The temporal ordering of chromatin remodeling complexes suggested by the above results is supported by studies on other promoters. Remodeling occurs indepen- 
dently of transcription of the SUC2 promoter in yeast (Hirschhorn et al. 1992); it can occur in the absence of activation of transcription by hormone receptors in mammals (Mymryk and Archer 1995; Wong et al. 1997), and precedes elongation of transcription through the human HSP70 gene (Brown and Kingston 1997). Although it is dangerous to make general conclusions from limited data, these studies together suggest that ATP-dependent remodeling of a nucleosome is an early step in the regulatory process, and that this precedes the binding of most transcription factors to nucleosomes. A clear and important exception to this is the binding by 'pioneer' activators, such as HNF3 or hormone receptors, which appear to be able to bind to nucleosomal DNA without the assistance of other factors to initiate the process of transcriptional activation (Cordingley et al. 1987; Pina et al. 1990; Cirillo et al. 1998).

The above studies of the HO gene suggest that acetylation events follow ATP-dependent remodeling. That this might be a more general phenomenon is suggested by biochemical studies that have shown very little effect of acetylation status on the function of ATP-dependent remodeling complexes, but have shown effects of remodeling on the rate of deacetylation (Imbalzano et al. 1994; Tong et al. 1998; Logie et al. 1999). It is likely, however, that ATP-dependent remodeling is not always required for changes in acetylation status. For example, activation of PHO5 requires HAT activity but does not require any known ATP-dependent remodeling activity (Gregory et al. 1998).

In the remainder of this review, we summarize the experiments that have been performed on ATP-dependent remodeling complexes, and propose a model that posits that the main role of these complexes is to create a fluid chromatin structure. We then summarize data related to the interactions between ATP-dependent remodeling complexes and acetyltransferase/deacetylase complexes, and further propose that the fluid chromatin structure created by remodeling complexes is locked into an activated or repressed state by acetylation/ deacetylation complexes together with other factors.

\section{Classes of remodeling complexes}

Many different ATP-dependent chromatin remodeling complexes have been identified; the two best studied classes of these complexes are the SWI/SNF family and the ISWI-based family. The ISWI family of complexes was originally identified through characterization of a Drosophila complex called nucleosome remodeling factor (NURF) that is able to remodel heat shock protein promoters in an ATP-dependent manner (Tsukiyama et al. 1994, 1995; Tsukiyama and Wu 1995). NURF was found to contain the protein ISWI as its central DNAdependent ATPase and three other proteins. The ISWI protein has a high degree of similarity with the DNAdependent ATPase subunits in the SWI/SNF family, and also with a growing list of proteins that appear to be involved in ATP-dependent remodeling (Fig. 1). At least two other ATP-dependent remodeling complexes in Dro-

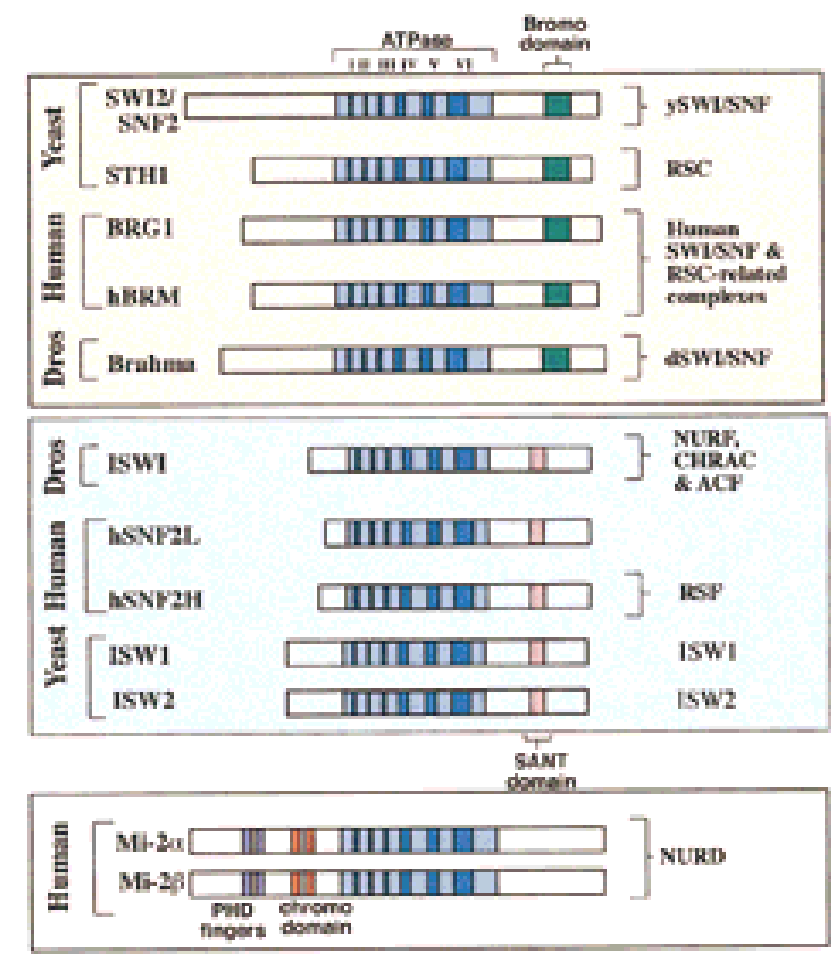

Figure 1. ATPase subunits of chromatin remodeling complexes; conserved domains are labeled. Protein names are shown at left; names of complexes at right; SWI/SNF family complexes are depicted in the pink box and ISWI-family complexes in the blue box.

sophila [chromatin remodeling and assembly complex (CHRAC) and ATP-utilizing chromatin assembly and remodeling factor (ACF)] also contain the ISWI protein but have different additional subunits and different characteristic functions (Fig. 2 and see below; Ito et al. 1997; VargaWeisz et al. 1997). Remodeling complexes containing apparent homologs of ISWI have been found in humans and yeast (LeRoy et al. 1998; Tsukiyama et al. 1999).

The first identification of members of the SWI/SNF family of complexes came from genetic screens in yeast and Drosophila for genes involved in regulation of transcription (in yeast) or developmental processes (in Drosophila) (for review, see Winston and Carlson 1992; Tamkun 1995). There are two SWI/SNF family members in yeast, SWI/SNF and RSC, that have 11 or more subunits with several subunits that are highly similar or identical (Cairns et al. 1994, 1996; Peterson et al. 1994). Characterization of human SWI/SNF complexes was made possible by the discovery of hBrm and BRG1, human homologs of the yeast DNA-dependent ATPase SWI2/SNF2 (Khavari et al. 1993; Muchardt and Yaniv 1993). The human SWI/SNF family of complexes was originally identified as a set of seven peptides that cofractionated with chromatin remodeling activity (Imbalzano et al. 1994; Kwon et al. 1994). Further refinements demonstrated that most human SWI/SNF complexes have eight or nine subunits, and that the precise composition of these complexes can vary with cell type (Wang 


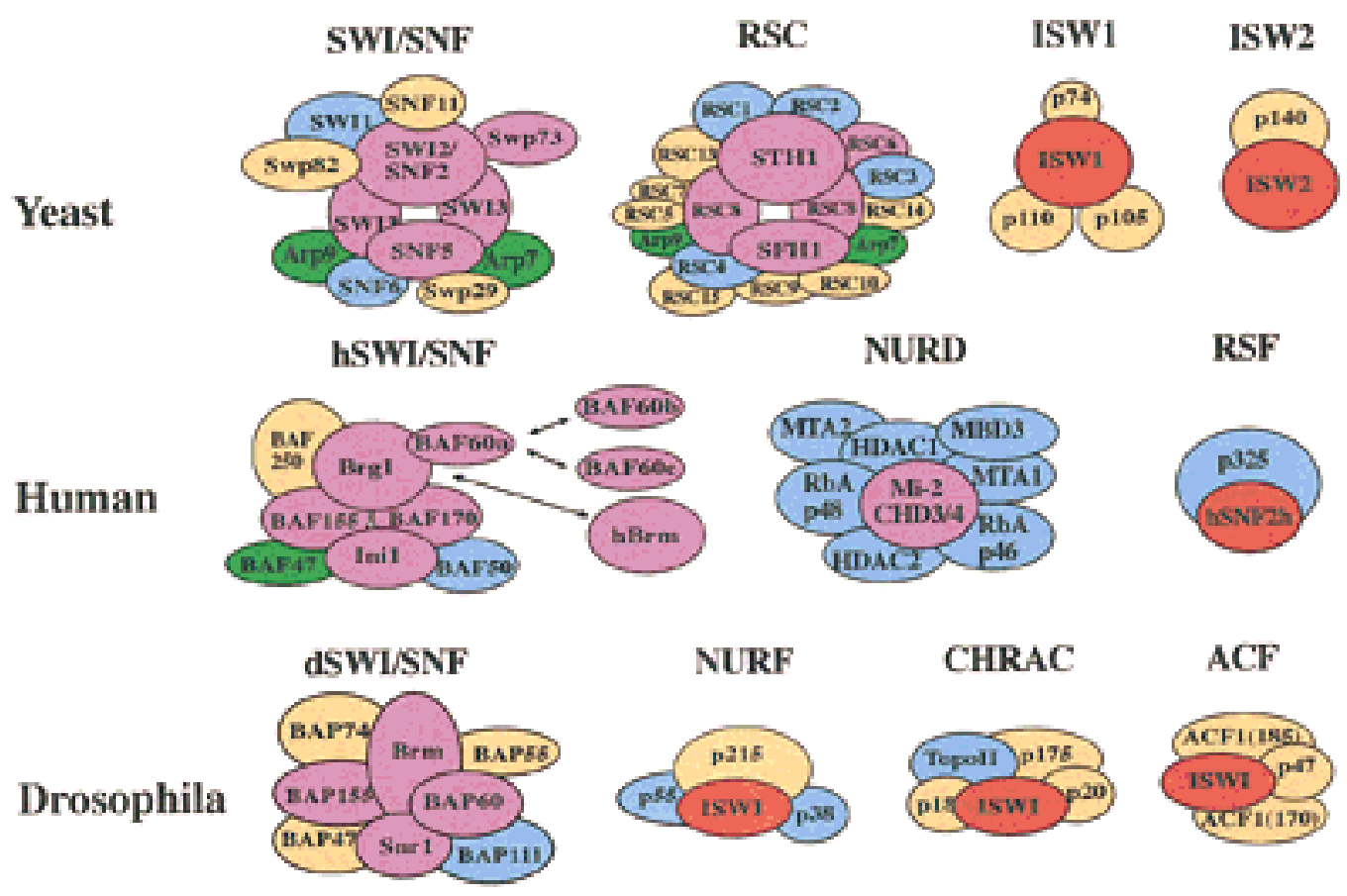

Figure 2. Composition of different ATP-dependent chromatin remodeling complexes. Conserved proteins in SWI/SNF family complexes are indicated in purple; ISWI homologs are indicated in red. Blue indicates proteins with known sequence, and green depicts actin-related proteins. Stoichiometries and direct contacts between proteins are speculative.

et al. 1996a,b). Recently, in Drosophila, the BRM complex has been purified and shown to contain homologs to the proteins that are common to the yeast and human SWI/SNF family complexes (Papoulas et al. 1998).

There are numerous other proteins with homologies to SWI2/SNF2 and to ISWI. The Mi-2 autoantigen has related isoforms that are homologous to SWI2/SNF2, and that have been identified as members of an ATP-dependent remodeling complex termed NURD (Fig. 1 and see below; Tong et al. 1998; Wade et al. 1998; Xue et al. 1998; Zhang et al. 1998b; Kim et al. 1999). This complex, although not characterized as thoroughly as the ISWI and SWI/SNF family complexes, appears to have some similar mechanistic characteristics. Given the number of proteins with homologies to the SWI2/SNF2 ATPase domains, it is likely that there will be many other ATPdependent remodeling complexes.

\section{Characteristic activities of ATP-dependent remodeling complexes}

Both the SWI/SNF and the ISWI families of complexes can perform several ATP-dependent reactions that each alter some characteristic of chromatin structure. The assays typically used to observe chromatin remodeling and our current understanding of the mechanisms of the remodeling reactions are summarized below.

DNase accessibility assays have been used to demonstrate that the SWI/SNF family of complexes can alter histone-DNA contacts in an ATP-dependent manner. DNase preferentially cleaves at positions of nucleosomal
DNA where the minor groove is facing away from the histone octamer. All tested members of the SWI/SNF family induce widespread changes in DNase cleavage patterns, suggesting a significant rearrangement of the path of the DNA on the octamer (Cote et al. 1994; Imbalzano et al. 1994; Cairns et al. 1996). In another assay, closed circular templates are assembled into nucleosomes, and then deproteinized following relaxation by topoisomerase. In the absence of SWI/SNF these templates contain one negative supercoil per nucleosome. Human or yeast SWI/SNF can dramatically change the topology of the template in an ATP-dependent manner, consistent with a SWI/SNF induced change in DNA path (Kwon et al. 1994; Wilson et al. 1996). SWI/SNF can also substantially increase restriction enzyme access and DNase access to DNA assembled within nucleosomal arrays (Owen-Hughes et al. 1996; Logie and Peterson 1997), and can increase binding of transcription factors to mononucleosomes and arrays (Cote et al. 1994; Imbalzano et al. 1994; Kwon et al. 1994; Owen-Hughes and Workman 1996).

The SWI/SNF family of complexes works catalytically as demonstrated by the ability of SWI/SNF to remodel nucleosomal arrays or mononucleosomes when less than one complex is present per nucleosome. Catalytic action on arrays of nucleosomes was also shown by demonstrating that yeast SWI/SNF could repeatedly induce remodeling upon addition of non-remodeled template to the reaction (Logie and Peterson 1997). The remodeled state, as detected by the assays described above, is stable after ATP is removed from the reaction under some, but not all, experimental conditions (Imbalzano et al. 1996; Lo- 
gie and Peterson 1997; Cote et al. 1998). A direct demonstration of stable remodeling was provided by the isolation of a distinct remodeled species following action of SWI/SNF or yeast RSC (remodel the structure of chromatin) on mononucleosomal substrates (Lorch et al. 1998; Schnitzler et al. 1998). This remodeled species retains the full complement of histones and DNA. Furthermore, in most cases the remodeled state can revert back, albeit slowly, to a standard nucleosomal state in the absence of SWI/SNF action. This reverse reaction implies that histones remain associated with DNA in the remodeled state as there is no detectable nucleosome formation by free histones and DNA under the same conditions.

Under certain conditions yeast RSC or SWI/SNF can also transfer histones. Yeast RSC will transfer histones from a subset of genomic chromatin to a labeled fragment of acceptor DNA (Lorch et al. 1999). Not all nucleosomes appear to be good substrates for histone transfer by RSC, however, probably because different nucleosomes on genomic DNA have different stabilities depending on how compatible the DNA sequence is with nucleosome formation. In a related reaction, yeast SWI/ SNF can promote transfer of histones to acceptor proteins from nucleosomes that are bound by GAL4 (OwenHughes and Workman 1996).

The SWI/SNF family is therefore capable of performing two different classes of remodeling reactions; one class creates a remodeled species that maintains all of the components of the nucleosome, and the second class results in physical transfer of histones. Histone transfer cannot account for SWI/SNF-induced changes in DNase accessibility of mononucleosomes or the changes in topology and restriction enzyme access on nucleosomal arrays, as these reactions are known to occur under situations that maintain the presence of histones. Both classes of reactions might use a similar intermediate; an 'activated' complex containing both RSC and a partially disrupted nucleosome has been argued to be the key intermediate in forming a stable remodeled nucleosome (via release of the intact remodeled nucleosome) and also in histone octamer transfer (via transfer of the histones from this activated complex to acceptor DNA) (Lorch et al. 1999).

In contrast to the SWI/SNF family of complexes, where to date every complex displays the same set of characteristic activities, individual ISWI-family remodeling complexes display marked differences in activities that presumably reflect differences in the details of their mechanism. One characteristic activity of a large subset of ISWI complexes is the ability to order a disordered array of nucleosomes. Nucleosomes that have been deposited onto long pieces of DNA either by salt dialysis or by the action of histone assembly proteins form irregularly spaced arrays. Several ISWI-family complexes are able to use the energy of ATP hydrolysis to create an ordered array with uniform spacing between nucleosomes (Varga-Weisz et al. 1995; Ito et al. 1997; Tsukiyama et al. 1999). These reactions require, in addition to the ISWI-based complex, protein(s) such as NAP1 that may act as histone acceptors. The exent of the ordering and the spacing between nucleosomes varies with different complexes. Several of the complexes capable of performing this function contain different proteins in addition to an ISWI family member; these other proteins might have an integral role in the mechanism of nucleosome spacing.

NURF, the founding member of the ISWI family, was originally discovered as an ATP-dependent activity that can promote the ability of the GAGA regulatory factor to remodel local chromatin structure (Tsukiyama et al. 1994; Tsukiyama and Wu 1995). The ATP-dependent activity presumably remodels all of the nucleosomal template and promotes binding by GAGA to specific sequences. Binding by GAGA then fixes a remodeled state of the adjacent chromatin. A yeast complex called ISW1 can also perform this function, however other complexes such as yeast ISW2 and CHRAC cannot (Varga-Weisz et al. 1997; Tsukiyama et al. 1999). Thus, complexes (e.g., CHRAC) that can move nucleosome position to allow regularly spaced arrays are not able to function in a manner that facilitates formation of a GAGA-induced remodeling event, suggesting potential mechanistic differences between these two processes. A related reaction that is performed by yet another set of ISWI family complexes is the induction of sensitivity to restriction enzyme cleavage on nucleosomal arrays. CHRAC, which does not induce GAGA remodeling, can perform this function whereas NURF cannot. The activities of the ISWI family, like SWI/SNF, are believed to be catalytic because these complexes are usually fully active at considerably less than one complex per nucleosome.

It has been proposed that each of the above activities of the ISWI family of complexes is based on the basic ability to loosen histone-DNA contacts in a manner that allows the nucleosome to move (Varga-Weisz et al. 1995; Ito et al. 1997; Alexiadis et al. 1998; Tsukiyama et al. 1999). Direct information on the ability of these complexes to move nucleosomes has come from experiments that start with DNA fragments that have nucleosomes at defined positions (Hamiche et al. 1999; Langst et al. 1999). Addition of either NURF or CHRAC caused the nucleosomes on these templates to slide along the DNA fragment in an ATP-dependent manner. The final position of these nucleosomes appeared to reflect the thermodynamic stability of those positions in the absence of remodeling agent (although see below).

It appears that the ISWI protein forms an important part of the catalytic core of this family of complexes. Bacterially produced ISWI protein can promote uniform spacing of nucleosomal arrays, remodeling of promoter chromatin in conjunction with GAGA factor, and movement of nucleosomes (Corona et al. 1999; Langst et al. 1999). In the full complexes, other proteins play mechanistic roles that alter ISWI's activities. ISWI and CHRAC differ in how they move nucleosomal position. ISWI moves a nucleosome towards the end of a short fragment, and CHRAC (which includes ISWI, topoisomerase II, and three other proteins) moves a nucleosome towards the center of the same fragment (Langst et al. 1999). This may reflect a difference in the mechanism by which 
movement is catalyzed, or it may reflect the presence of an end-binding activity in CHRAC that excludes the more thermodynamically stable position where a nucleosome is associated with a fragment end. ACF has recently been shown to have a second subunit (ACF1) that significantly increases the ability of ISWI to participate in assembly of appropriately spaced nucleosomes (Ito et al. 1999). This latter behavior is similar to that seen in the SWI/SNF family; here, BRG1 is capable of inefficient ATP-dependent remodeling that is enhanced by the addition of three other subunits of human SWI/ SNF (Phelan et al. 1999).

Although the SWI/SNF and ISWI families perform similar types of ATP-dependent remodeling reactions, mechanistic differences between the two are suggested by the significant differences in substrate specificity. Remodeling by NURF requires histone tails, whereas remodeling by human and yeast SWI/SNF does not (Georgel et al. 1997; Guyon et al. 1999; Logie et al. 1999). The ATPase activity of the SWI/SNF complexes is stimulated equally well by nucleosomal DNA and naked DNA, but the ATPase activity of several ISWI complexes is stimulated only by nucleosomal DNA (Laurent et al. 1993; Cote et al. 1994; Tsukiyama and Wu 1995). These characteristics suggest that the SWI/SNF and ISWI families interact with different portions of the nucleosome.

Despite the apparent mechanistic differences between the two families of complexes, it appears that both families are similar in their use of the energy of ATP hydrolysis to alter nucleosome conformations or nucleosome locations. A significant, unanswered question concerns which of these reactions is responsible for the biological effects of remodeling in vivo. Techniques are not yet available to determine whether any or all of the events described above occur in vivo. We argue below that it is not necessarily any of these individual reactions, but rather the ability of the SWI/SNF and ISWI families to catalyze interconversions of nucleosome structure or location, that is the key to regulatory function.

\section{Catalyzing chromatin fluidity}

All of the data described in the previous section are consistent with a simple general hypothesis for the function of ATP-dependent remodeling complexes: these complexes function as classic enzymes and use the energy of ATP hydrolysis to lower the activation barriers between different nucleosomal states (Fig. 3). These chromatin states might differ in either the position of nucleosomes and/or the conformation of the nucleosome. Normally, the different chromatin states would interconvert slowly in the absence of remodeling complexes. The proposal here posits that the action of ATP-dependent remodeling complexes does not modify nucleosomes in a manner that alters the thermodynamic stability of any product. Rather, these complexes increase the rate of interconversion between different states to the extent that these states can be accessed in a time frame that is compatible with biological regulatory processes.

This proposal is best illustrated by the activity of SWI/ SNF on mononucleosomes. As described above, human SWI/SNF and RSC can generate a stable remodeled species by acting on standard mononucleosomes (Fig. 3). It was initially surprising that these complexes were also able to use the energy of ATP hydrolysis to regenerate the standard conformation from the remodeled species (Lorch et al. 1998; Schnitzler et al. 1998). Furthermore, the final ratio of the standard and remodeled conformations after action of SWI/SNF appears to be the same whether the standard state or the remodeled state is the starting point.

These results are explained most simply by the previous proposal that the conformational transition proceeds via an activated intermediate state that is bound to SWI/ SNF (Lorch et al. 1998; Schnitzler et al. 1998). The intermediate can collapse stochastically to the remodeled or standard state. The ratio of remodeled to standard state after multiple rounds of SWI/SNF activity will then be determined primarily by the relative rates at

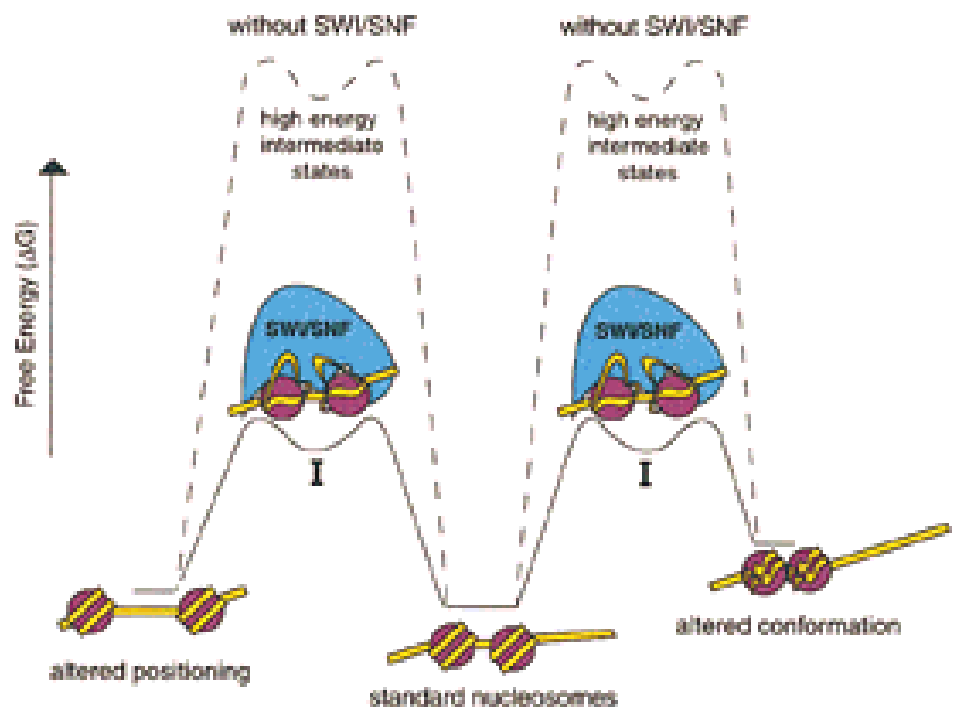

Figure 3. A model for SWI/SNF function. The effect of SWI/SNF is depicted using highly schematic freeenergy profiles. In the absence of SWI/SNF, the interconversion between nucleosome states is slow due to the high energy of intermediate states (broken line). SWI/SNF is proposed to use the energy of ATP hydrolysis to facilitate creation of an activated intermediate (I) that has loosened histone-DNA contacts. This increases the rate of interconversion between a standard (center) and remodeled (right) nucleosomal state. The same activated intermediate might also collapse to a state with altered nucleosomal positions (left). The steps of ATP binding and hydrolysis are not shown for clarity; it is not known how ATP hydrolysis is coupled to the remodeling reaction. Similar models have been proposed for ISWI-based complexes (Alexiadis et al. 1998; Ito et al. 1997, 1999; Hamiche et al. 1999; Tsukiyama et al. 1999). 
which the intermediate partitions to either state. The rate of equilibration between the two species in the absence of remodeling activity will also contribute to the final ratio; however, this is likely to occur slowly compared to a physiological time scale. Thus, by stabilizing a high-energy intermediate state, SWI/SNF can reduce the activation energy for interconversion between two chromatin states (Fig. 3). A similar (or perhaps identical?) intermediate state is proposed to lead to histone transfer by the RSC complex (Lorch et al. 1999). In this case, formation of the intermediate would facilitate exchange of the sequence of DNA that is bound by the histones. This is also an enzymatically reversible process, as the DNA sequences that are used as donor and acceptor in the transfer can be interchanged.

ISWI-based reactions have previously been postulated to go through an intermediate with weakened histoneDNA contacts (Ito et al. 1997; Alexiadis et al. 1998; Hamiche et al. 1999). Although it appears from the differences in substrate specificity that this intermediate is created by different contacts between the ISWI complexes and the nucleosome than are used by SWI/SNF, the concept of creating an activated state that can collapse into states with different nucleosome positions is similar to that proposed for the SWI/SNF family. It is known that nucleosomes can shift positions in an unaided fashion in solution (Meersseman et al. 1992), and it is possible that the ISWI complexes weaken histoneDNA contacts in a manner that increases the rate of this reaction. Thus, different contacts and different mechanisms can be used by the ISWI and SWI/SNF families to increase the rate of different types of transitions in chromatin structure. In general, each of the different ATPdependent remodeling complexes might be able to catalyze a subset of the possible transitions that chromatin structure can make.

This hypothesis has a profound consequence for the role that ATP-dependent remodeling complexes might play in regulation. If the main purpose of these complexes is to catalyze transitions between chromatin states having different structures, then the ATP-dependent remodeling complexes themselves do not specify whether the endpoint that is reached is an activated or a repressed chromatin state. These complexes simply increase the rate at which different chromatin states can be formed. If factors are present that stabilize a chromatin structure that represses transcription, these factors might remove that structure from the remodeling reaction. In this instance, the remodeling complexes will help drive the chromatin conformation onto a repressed state. Conversely, if factors that bind the activated state are present, then the remodeling complexes would drive the structure toward an active state (Fig. 4). Targeting of ATP-dependent remodeling activities to a gene might provide a mechanism that increases the rate of transition between an active chromatin configuration and a repressed chromatin configuration; the direction of the transition, however, would be determined by other factors.

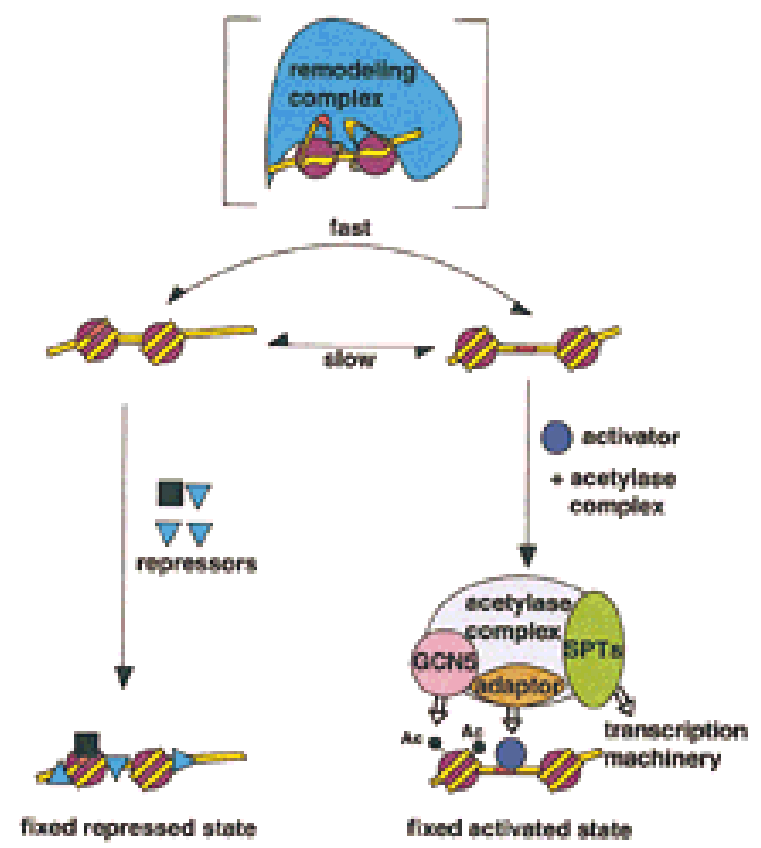

Figure 4. A hypothetical scheme for coordination between ATP-dependent remodeling activities and acetylases/deacetylases. The ATP-dependent remodeling complex is proposed to increase the rate of interconversion between different chromatin states. Activating complexes and acetyltransferases are proposed to preferentially bind activated states, and thus "fix" those chromatin configurations. Similarly, repressors are proposed to preferentially bind and fix repressed states. Although there is no direct evidence, it is formally possible that certain remodeled conformations will not be recognized as substrates by the remodeling complex, resulting in fixing these conformations in the absence of additional factors.

\section{Targeting and regulation of remodeling activities}

Experiments performed in several systems have suggested that SWI/SNF family complexes can be targeted to specific regions of the genome. The constitution of these complexes indicates that significant portions of each complex might play a role in targeting. These complexes are quite large ( 2 MD), and it is clear that this size is not absolutely required to perform the basic remodeling reaction. For example, many of the enzymatic reactions of human SWI/SNF can be performed by four of the eight total subunits (Phelan et al. 1999). Regulation of remodeling activity would appear to be an attractive possible role for the remainder of the proteins in these complexes.

Studies using chromatin immunoprecipitation techniques in yeast demonstrate targeting of SWI/SNF. This complex is bound to the $H O$ gene only at specific times during cell cycle, and this binding does not occur when the activator SWI5p is not present (Cosma et al. 1999). In studies of the HTA1 promoter, binding of SWI/SNF requires the presence of the HIR proteins (DiMova et al. 1999|. Thus, in both cases, sequence-specific DNA-binding proteins are required to localize yeast SWI/SNF to promoters, implying that these DNA-binding proteins 
target SWI/SNF. In mammals, several steroid receptors have been shown to interact with various components of SWI/SNF, and more directly glucocorticoid receptor has been shown to target ATP-dependent remodeling activity to a mononucleosome (Fryer and Archer 1998; Yoshinaga et al. 1992; Ostlund et al. 1997).

There is also considerable evidence for targeting of ATP-dependent remodeling complexes containing the Mi-2 protein. These Mi-2-based complexes constitute a family distinct from the ISWI and SWI/SNF complexes, and appear to be generally involved in repression as they are associated with transcriptional repressors and contain the histone deacetylase HDAC1. However, Mi-2based complexes perform many of the same remodeling activities as the SWI/SNF family of complexes. Mi-2 has been shown to be physically associated with the Ikaros family of sequence-specific DNA-binding proteins in $\mathrm{T}$ cells, and to colocalize with Ikaros in vivo (Kim et al. 1999|. In Drosophila, dMi-2 associates with the repressor Hunchback (Kehle et al. 1998). In addition, numerous DNA-binding repressors are known to associate with HDAC1, and presumably some of these also associate with Mi-2. Thus several different DNA-binding proteins that play a repressive role contact $\mathrm{Mi}-2$, suggesting that Mi-2 and remodeling complexes containing Mi-2 are targeted via these interactions.

If, as we propose above, remodeling activities do not specify a regulatory endpoint, then why are these activities targeted? If the sole function of these complexes is to create fluid chromatin environments, then, in theory, regulatory processes might work appropriately whether or not there is any targeting of the complexes. There are, however, two compelling reasons for targeting these activities. First, these complexes catalyze the hydrolysis of 50-200 ATP molecules per complex per minute, so indiscriminate activity would waste large amounts of energy. Second, a simple mechanism of repression would be to take advantage of the intrinsic repressive nature of a standard nucleosome. The kinetic barrier to rearranging nucleosome positions and/or conformations in standard chromatin structures might be important in maintaining a repressed state. Keeping remodeling complexes away from certain segments of the genome might play a regulatory role simply by maintaining the default state of the chromatin.

There is good evidence that ATP-dependent remodeling activities are broadly inactivated at stages of the cell cycle where chromatin is held in a condensed state, consistent with a model in which the activities of these complexes are modulated to help maintain the kinetic barrier to chromatin rearrangement. When cells enter mitosis, the human SWI/SNF complexes are excluded from chromatin and their remodeling activities are inactivated by phosphorylation (Muchardt et al. 1996; Sif et al. 1998). The complexes are dephosphorylated and reactivated as cells exit mitosis, concomitant with chromatin decondensation. In resting $\mathrm{T}$ cells, the chromosome is condensed and SWI/SNF complexes are also excluded from chromatin (Kim et al. 1999; Zhao et al. 1998). Upon stimulation of T cells, SWI/SNF associates with chro- matin at the time of chromatin decondensation. Although there is not yet any experimental evidence that activation of SWI/SNF complexes has a causal role in facilitating decondensation of chromatin in either of these instances, it is an attractive hypothesis.

Thus, ATP-dependent remodeling complexes can be specifically targeted to genes via interactions with sequence-specific DNA-binding proteins, and can also be regulated in a more general way by inactivation and/or relocalization. The fact that mechanisms exist that broadly inactivate entire classes of remodeling complexes at certain stages of cell growth emphasizes the general importance of remodeling. ATP-dependent remodeling is likely to affect many different types of regulatory processes that occur on chromatin (e.g., replication, recombination, repair), and thus the regulation of these activities is likely to be important in regulating nuclear architecture. It is intriguing that all SWI/SNF family complexes have recently been shown to contain actin-related proteins (ARPs) that do not appear to be required for any catalytic remodeling functions (Cairns et al. 1998; Peterson et al. 1998; Zhao et al. 1998). Genetic studies using yeast demonstrate that these proteins are required for the action of the SWI/SNF family in vivo, and it has been proposed that these proteins may play a general role in modulating the activities of SWI/ SNF family members, perhaps by helping to recruit SWI/ SNF to chromatin.

\section{Transcriptional regulation by ATP-dependent remodelers}

Despite their prominence as potential regulators of various transcriptional processes, there have been relatively few experiments that have directly examined the effects of ATP-dependent remodeling complexes on the efficiency of transcription. In vitro experiments of this type have been hampered by the significant technical difficulties involved in reconstituting transcription from components that are free of remodeling activities, and then characterizing the effects of adding back purified remodeling activities. Two ISWI family complexes, NURF and remodeling and spacing factor (RSF), have been shown to assist transcriptional activation by GAL4 fusion proteins on test promoters that were assembled into nucleosomes (Mizuguchi et al. 1997; LeRoy et al. 1998). In both cases, the majority of transcriptional activation by the GAL4 fusion protein was dependent upon addition of these remodeling complexes. In similar studies, activation of a promoter by the activator EKLF was dependent on a human SWI/SNF family complex (Armstrong et al. 1998). These experiments demonstrate that the different remodeling complexes are able to participate functionally in the overall transcriptional activation process, and therefore dramatically extend previous experiments that had shown the potential of these complexes to assist in various isolated steps of the transcriptional activation process.

Although these in vitro experiments illustrate the mechanistic capabilities of remodeling complexes, ge- 
netic experiments are required to determine which complexes are actually necessary for transcriptional regulation of specific genes in vivo. To date, the only remodeling complex that has been strongly implicated in regulation of specific promoters in vivo is SWI/SNF. The original data concerning genetic analyses of SWI/SNF mutations on individual promoters have been reviewed (Winston and Carlson 1992; Carlson and Laurent 1994; Kingston et al. 1996). Recent studies that have used microarrays to examine how regulation changes across the entire yeast genome in SWI/SNF mutants yielded the surprising finding that SWI/SNF appears to act more frequently in repression events than in activation events (Holstege et al. 1998). Whether these effects are direct or indirect effects of SWI/SNF is not clear; however, the large number of genes that require SWI/SNF for an appropriately repressed state suggests that at least some of these repressive effects might be direct. In addition, yeast RSC and human SWI/SNF have been implicated in repression of the CHA1 and c-fos genes, respectively (Moreira and Holmberg 1999; Murphy et al. 1999). These studies are consistent with the hypothesis proposed above, in that they suggest that the involvement of SWI/ SNF remodeling at a promoter does not by itself specify whether the final chromatin state is activated or repressed; rather, ATP-dependent remodeling is used as a tool to facilitate formation of the required chromatin state.

It is clear from further genetic studies in S. cerevisiae that different ATP-dependent remodeling complexes play very different biological roles. Null mutations in components in the RSC complex cause lethality, indicating a clear phenotypic difference between mutations in RSC and SWI/SNF (Cairns et al. 1996; Cao et al. 1997; Du et al. 1998). Null mutations in genes encoding the central ATPase subunits of the ISW1 or ISW2 complexes do not cause obvious phenotypes, but there are synthetic phenotypes between mutations that abolish ISW1, ISW2, and a presumed complex based around the ATPase CHD (Tsukiyama et al. 1999). The different roles of these ATP-dependent remodeling complexes in vivo presumably reflects both the difference in the types of remodeling reactions that these complexes can perform as well as differences in targeting and regulation of the complexes.

\section{Maintenance of activation}

Recent studies demonstrate that SWI/SNF must be continually present to maintain the activated state of several different genes (Biggar and Crabtree 1999; Sudarsanam et al. 1999). If, as is hypothesized above, SWI/SNF action is primarily used to faciliate interconversions between repressive and active chromatin states and the active state is fixed by other factors, then why would SWI/SNF be continually required to keep a gene in an active state? It is possible that inactivation of these genes in the absence of SWI/SNF occurs in a reasonably rapid, stochastic fashion. For example, the activated chromatin state might be unstable, and might decay to a repressed structure at a rapid rate, and SWI/SNF might be required to enhance the rate at which the active state is regained. (The studies that have shown a stable remodeled state for nucleosomes in vitro have mostly been performed with mononucleosomal templates; the rate of decay from any remodeled state in vivo in the context of an array of nucleosomes, and in the presence of cellular components, is not known.) Alternatively, it is also possible that SWI/SNF plays a more direct role in activation in this instance such as forming part of the physical bridge between the activator and RNA polymerase that is required to maintain the active state.

\section{Acetylation as a mechanism of fixing chromatin states}

Acetylation and deacetylation complexes have the obvious, and likely important, difference from remodeling complexes in that they cause a covalent modification of the nucleosome. Acetylation is usually (but not always; see Bresnick et al. 1990) correlated with activation, and deacetylation is usually correlated with repression. If ATP-dependent remodeling is used primarily to interconvert different chromatin structures without regard to outcome, then acetylation or deacetylation might be an essential part of the process that alters the thermodynamic stability of a given chromatin structure, and thereby helps to fix the structure into either an active or a repressed state.

Acetylation and deactylation complexes have been extensively reviewed recently (Grunstein 1997; Hartzog and Winston 1997; Kadonaga 1998; Kuo and Allis 1998; Mizzen and Allis 1998; Struhl 1998; Workman and Kingston 1998); the discussion below focuses on the SptAda-Gcn5-acetyltransferase (SAGA) HAT complexes and the interactions between this complex and the SWI/ SNF complex. Numerous different HATs have been identified, primarily through their direct contact with sequence-specific activators. Several of these proteins are also capable of acetylating other regulatory factors, and it is not completely clear which HATs have histones as their primary substrate in vivo. Genetic and chromatin immunoprecipitation studies have been used to show that GCN5 does use histones as a biologically relevant target (Candau et al. 1997; Kuo et al. 1998; Zhang et al. 1998a). GCN5 is part of a large complex called SAGA that also contains the products of numerous other genes that were originally identified in screens for effects on transcriptional activation (these include members of the SPT family and the ADA family) (Grant et al. 1997; Roberts and Winston 1997). This complex is conserved evolutionarily [the p300/CBP-associated factor (PCAF) complex in humans is one apparent homolog] and also contains TAF proteins in addition to the aforementioned families of proteins (Grant et al. 1998; Ogryzko et al. 1998). There are several multisubunit HAT complexes in yeast, and it appears that these other complexes are also conserved evolutionarily (Grant et al. 1997; Ikeda et al. 1999|. Thus, as has been seen with ATP-dependent remodeling complexes, different families of HAT complexes will likely play distinct biological roles. 


\section{Multiple functions of SAGA}

The HAT capability of SAGA is known to be important for its function. A large series of point mutations has been created in GCN5 that severely cripple acetyltransferase activity (Candau et al. 1997; Kuo et al. 1998). These point mutations impair activation of transcription, implying that acetylation is a key aspect of GCN5 function. In addition, mutations that change acetylated residues to glutamine (thereby mimicking a constitutively acetylated state) largely bypass a need for GCN5 in activation (Zhang et al. 1998a).

Further genetic studies demonstrate, however, that there are likely to be other mechanisms for SAGA action in addition to acetylation. Mutations in all components of SAGA cause synthetic phenotypes when combined with mutations in the genes that encode SWI/SNF, implying a related function for SAGA and SWI/SNF (Pollard and Peterson 1997; Roberts and Winston 1997). Deletions of either SPT20 or SPT7 cause more severe synthetic phenotypes with mutations in SWI/SNF components than deletion of GCN5, implying that SPT20 and SPT7 play a role in SAGA that is independent of GCN5 function, and that presumably is independent of acetyltransferase activity (Roberts and Winston 1997; Sterner et al. 1999). Mutation of SPT3 also gives relatively mild phenotypes but does not affect acetyltransferase function. However, in an spt3 gcn5 double mutant, the rest of the SAGA complex remains physically intact but the strain has severe phenotypes that appear equivalent to mutations that completely disrupt the SAGA complex. Thus, GCN5 and SPT3 each play a functionally distinct role in SAGA.

These studies contrast with genetic studies on ATPdependent remodeling activities, in which point mutations that eliminate the ability to hydrolyze ATP in several complexes have the same phenotype as a null in the ATPase subunit (Laurent et al. 1993; Tsukiyama et al. 1999). In addition, mutations in other genes in the SWI/ SNF family of complexes have never been found to be more severe than null mutations in the ATPase subunits of these complexes. [There are ATP-dependent remodeling activities that do have multiple roles (see below); however, some ATP-dependent remodeling activities appear to have that property as their primary mode of action.]

In vitro studies support a direct role for acetylation in transcriptional activation on nucleosomal templates (Sheridan et al. 1997; Steger et al. 1998; Ikeda et al. 1999). Acetylation can increase the ability of various regulatory proteins to bind to specific sequences (Lee et al. 1993; Vettese-Dadey et al. 1994). Increased acetylation directed by SAGA (or other HAT complexes) increases the rate of activator-dependent transcription in a manner that can require the presence of acetyl-CoA, and, by inference, acetylation activity (Ikeda et al. 1999). Thus, acetylation can aid the binding and function of proteins involved in activation. Acetyltransferase activity is not absolutely required for transcriptional activation on all nucleosomal substrates, however, as activation by vari- ous activators is frequently seen in vitro in the absence of acetyl-CoA on nucleosomal templates that are largely deacetylated (e.g., Workman et al. 1991; Kamakaka et al. 1993; Kraus and Kadonaga 1998).

As described above, genetic analysis implies that SAGA assists in other steps of the transcription reaction. Biochemical studies suggest that SAGA might form a physical bridge between activators and the general transcription machinery, and therefore might be involved in stabilizing the transcription complex on a promoter (Sterner et al. 1999). SAGA is known to bind to TBP and also to activators (Utley et al. 1998). Thus, SAGA may increase transcription both by modifying the template to a more accessible state and also by recruiting transcription factors (see Fig. 3).

\section{A model for cooperation between SAGA and SWI/SNF}

SAGA and SWI/SNF appear to work in concert to activate several genes (Biggar and Crabtree 1999; Sudarsanam et al. 1999|. One possible mechanism for cooperation between these complexes is that SWI/SNF creates a fluid chromatin environment, which is then locked into an activated state by the combined actions of the different components of SAGA. Thus, SWI/SNF action on a promoter might be continually scrambling nucleosome position and/or conformation to any number of different states. Each time a state is reached that is compatible with activator/transcription factor binding, SAGA will help fix the activated state by promoting binding to that state and thereby removing it from the reaction (Fig. 4). According to this hypothesis, SAGA and other components of the transcription machinery could fix an active chromatin state in the absence of SWI/SNF function, the reaction would simply take longer due to the slow intrinsic rates of interconversion between chromatin states. Analogously, the components of the transcription machinery could fix a chromatin state in the absence of SAGA function, but the fixed state would be less stable than if SAGA were present. Thus, SAGA and SWI/SNF would play very different roles that would have the common goal of efficiently establishing an active chromatin state. This model is consistent with the genetic and molecular studies, in that it provides a mechanistic explanation for why SWI/SNF and SAGA mutations can cause synthetic lethality and for why these complexes appear to have redundant functions on certain promoters (Roberts and Winston 1997; Biggar and Crabtree 1999; Sudarsanam et al. 1999).

SAGA has several characteristics that are well suited to a complex involved in fixing a specific chromatin state. It can acetylate histones-this is known to promote binding by components of the transcription machinery-and also appears to alter the thermodynamic stability of nucleosomes. The histone amino-terminal tails have a crucial role in stabilizing nucleosomal arrays (Fletcher and Hansen 1995; reviewed in Workman and Kingston 1998), and modification of the tails is expected to significantly affect the stability of nucleosome structure via effects on internucleosome contacts. SAGA can 
also interact physically with both activators and general transcription factors and thereby directly facilitate their binding (Fig. 4). Therefore, all known characteristics of SAGA function are consistent with an ability to stabilize a chromatin state that is bound by both activators and general transcription factors, and is thus an active state. There is support for this notion in the recent characterization of the HO promoter. Cross-linking of SAGA and acetylation of this promoter are dependent on SWI/SNF action, and the activator SWI4 and SAGA bind at approximately the same time (Cosma et al. 1999). Thus, SWI/SNF might increase the frequency of formation of a chromatin configuration on $\mathrm{HO}$ that is compatible with the binding of SWI4 and SAGA, and SAGA action (both acetylation and other activities) might help stabilize this conformation.

\section{Deacetylases and ATP-dependent remodeling complexes}

The combined action of ATP-dependent remodeling activities and changes in acetylation status is seen even more dramatically with deacetylase complexes. One of the primary deacetylases in mammalian cells, HDAC1, has been found to reside in a complex (termed NURD) that has ATP-dependent remodeling activity (Tong et al. 1998; Wade et al. 1998; Xue et al. 1998; Zhang et al. 1998b). The central DNA-dependent ATPase of this complex, Mi-2, has sequence similarity with SWI2/ SNF2, and complexes that contain Mi-2 perform some of the same remodeling activities as the SWI/SNF family. Remodeling activity stimulates the ability of NURD to deacetylate test templates (Tong et al. 1998), so in this instance ATP-dependent remodeling might be necessary to promote a deacetylation event that is primarily associated with repression.

Genetic studies in yeast on the RPD3 deacetylase, which is homologous to HDAC1, indicate that deacetylation is an important portion of the repressive capability of this protein but is not the only mechanism used to repress transcription (Rundlett et al. 1996, 1998; Kadosh and Struhl 1998). Point mutations that block deacetylase function decrease, but do not eliminate, the repressive function of RPD3 (Kadosh and Struhl 1998), suggesting that RPD3 has functions apart from deacetylation that are involved in establishing a repressed state. Complexes containing RPD3 appear to be targeted directly by sitespecific repressors such as UME6 (Kadosh and Struhl 1997) analogous to the targeting of the HDAC1 complexes discussed above.

The mechanisms used by these deacetylase complexes to establish a repressed state have not been characterized at the same level as the mechanisms used in activation. It is not known whether these deacetylase complexes have direct contacts with the general transcription machinery, or whether they contact complexes that might stably bind and 'coat' chromatin (e.g., in a manner similar to that proposed for the SIR complex; Hecht et al. 1995; Renauld et al. 1993) to establish a physically repressed state. By analogy to the mechanism proposed above for SAGA function, it is possible that deacetylase complexes both deacetylate nucleosomes to promote a more stable structure and recruit proteins that bind to the deacetylated template to stabilize a repressive chromatin state. ATP-dependent remodeling might be involved both in increasing the rate at which deacetylation occurs and in facilitating the transition to a chromatin structure that is compatible with binding by repressive complexes.

\section{Conclusions}

It is apparent that chromatin structure is dynamic and that structural changes in chromatin are highly regulated. Current data suggest that one role of ATP-dependent remodeling complexes is to use the energy of ATP hydrolysis to increase the rate at which different structures interchange. These complexes thus make chromatin more fluid. Mechanisms must also exist to fix genes in an active or a repressed state; here, acetylation and deacetylation complexes might be key players. Thus, both the rate at which a chromatin remodeling event can occur and the thermodynamic stabilities of the end products are essential to regulation. It appears as if nature has devised large families of complexes to govern both aspects of chromatin dynamics, allowing an exquisite regulation of chromatin structures.

\section{Acknowledgments}

We thank S. Levine, C. Peterson, D. Reinberg, G. Schnitzler, F. Winston, J. Workman, and members of our laboratory for comments; M. Phelan for Figure 1; and S. Sif for Figure 2. Work in our laboratory was supported by grants from Damon RunyonWalter Winchell Foundation (G.J.N.), the National Institutes of Health and Hoechst AG (R.E.K.).

\section{References}

Alexiadis, V., P.D. Varga-Weisz, E. Bonte, P.B. Becker, and C. Gruss. 1998. In vitro chromatin remodelling by chromatin accessibility complex (CHRAC) at the SV40 origin of DNA replication. EMBO I. 17: 3428-3438.

Allfrey, V.G., R. Faulkner, and A.E. Mirsky. 1964. Acetylation and methylation of histones and their possible role in the regulation of RNA synthesis. Proc. Nat1. Acad. Sci. 51: 786794.

Armstrong, J.A., J.J. Bieker, and B.M. Emerson. 1998. A SWI/ SNF-related chromatin remodeling complex, E-RC1, is required for tissue-specific transcriptional regulation by EKLF in vitro. Cell 95: 93-104.

Biggar, S.R. and G.R. Crabtree. 1999. Continuous and widespread roles for the Swi-Snf complex in transcription. EMBO I. 18: 2254-2264.

Bresnick, E.H., S. John, D.S. Berard, P. LeFebvre, and G.L. Hager. 1990. Glucocorticoid receptor-dependent disruption of a specific nucleosome on the mouse mammary tumor virus promoter is prevented by sodium butyrate. Proc. Natl. Acad. Sci. 87: 3977-3981.

Brown, S.A. and R.E. Kingston. 1997. Disruption of downstream chromatin directed by a transcriptional activator. Genes \& Dev. 11: 3116-3121. 
Cairns, B.R., Y.-J. Kim, M.H. Sayre, B.C. Laurent, and R.D. Kornberg. 1994. A multisubunit complex containing the SWI1/ADR6, SWI2/SNF2, SWI3, SNF5, and SNF6 gene products isolated from yeast. Proc. Natl. Acad. Sci. 91: 19501954.

Cairns, B.R., Y. Lorch, Y. Li, M. Zhang, L. Lacomis, H. Erdjument-Bromage, P. Tempst, J. Du, B. Laurent, and R.D. Kornberg. 1996. RSC, an essential, abundant chromatin-remodeling complex. Cell 87: 1249-1260.

Cairns, B.R., H. Erdjument-Bromage, P. Tempst, F. Winston, and R.D. Kornberg. 1998. Two actin-related proteins are shared functional components of the chromatin-remodeling complexes RSC and SWI/SNF. Mol. Cell 2: 639-651.

Candau, R., J.X. Zhou, C.D. Allis, and S.L. Berger. 1997. Histone acetyltransferase activity and interaction with ADA2 are critical for GCN5 function in vivo. EMBO J. 16: 555-565.

Cao, Y., B.R. Cairns, R.D. Kornberg, and B.C. Laurent. 1997. Sfhlp, a component of a novel chromatin-remodeling complex, is required for cell cycle progression. Mol. Cell. Biol. 17: 3323-3334.

Carlson, M. and B.C. Laurent. 1994. The SNF/SWI family of global transcriptional activators. Curr. Opin. Cell Biol. 6: 396-402.

Cirillo, L.A., C.E. McPherson, P. Bossard, K. Stevens, S. Cherian, E.Y. Shim, K.L. Clark, S.K. Burley, and K.S. Zaret. 1998. Binding of the winged-helix transcription factor HNF3 to a linker histone site on the nucleosome. EMBO J. 17: 244-254.

Cordingley, M.G., A.T. Riegel, and G.L. Hager. 1987. Steroiddependent interaction of transcription factors with the inducible promoter of mouse mammary tumor virus in vivo. Cell 48: 261-270.

Corona, D.F., G. Langst, C.R. Clapier, E.J. Bonte, S. Ferrari, J.W. Tamkun, and P.B. Becker. 1999. ISWI is an ATP-dependent nucleosome remodeling factor. Mol. Cell 3: 239-245.

Cosma, M.P., T. Tanaka, and K. Nasmyth. 1999. Ordered recruitment of transcription and chromatin remodeling factors to a cell cycle- and developmentally regulated promoter. Cell 97: 299-311.

Cote, J., J. Quinn, J.L. Workman, and C.L. Peterson. 1994. Stimulation of GAL4 derivative binding to nucleosomal DNA by the yeast SWI/SNF complex. Science 265: 53-60.

Cote, J., C.L. Peterson, and J.L. Workman. 1998. Perturbation of nucleosome core structure by the SWI/SNF complex persists after its detachment, enhancing subsequent transcription factor binding. Proc. Natl. Acad. Sci. 95: 4947-4952.

DiMova, D., Z. Nackerdien, S. Furgeson, S. Eguchi, and M.A. Osley. 1999. A role for transcriptional repressors in targeting the yeast Swi/Snf complex. Mol. Cell 4: 75-83.

Du, J., I. Nasir, B.K. Benton, M.P. Kladde, and B.C. Laurent. 1998. Sth1p, a Saccharomyces cerevisiae Snf2p/Swi2p homolog, is an essential ATPase in RSC and differs from Snf/ Swi in its interactions with histones and chromatin-associated proteins. Genetics 150: 987-1005.

Felsenfeld, G., J. Boyes, J. Chung, D. Clark, and V. Studitsky. 1996. Chromatin structure and gene expression. Proc. Nat1. Acad. Sci. 93: 9384-9388.

Fletcher, T.M. and J.C. Hansen. 1995. Core histone tail domains mediate oligonucleosome folding and nucleosomal DNA organization through distinct molecular mechanisms. J. Biol. Chem. 270: 25359-25362.

Fryer, C.J. and T.K. Archer. 1998. Chromatin remodelling by the glucocorticoid receptor requires the BRG1 complex. Nature 393: 88-91.

Georgel, P.T., T. Tsukiyama, and C. Wu. 1997. Role of histone tails in nucleosome remodeling by Drosophila NURF. EMBO I. 16: 4717-4726.
Grant, P.A., L. Duggan, J. Cote, S. Roberts, J.E. Brownell, R. Candau, R. Ohba, T. Owen-Hughes, C.D. Allis, F. Winston, S.L. Berger, and J.L. Workman. 1997. Yeast Gen5 functions in two multisubunit complexes to acetylate nucleosomal histones: Characterization of an Ada complex and the SAGA (Spt/Ada) complex. Genes \& Dev. 11: 1640-1650.

Grant, P.A., D. Schieltz, M.G. Pray-Grant, D.J. Steger, J.C. Reese, J.R. Yates III, and J.L. Workman. 1998. A subset of TAF(II)s are integral components of the SAGA complex required for nucleosome acetylation and transcriptional stimulation. Cell 94: 45-53.

Gregory, P.D. and W. Horz. 1998. Life with nucleosomes: chromatin remodelling in gene regulation. Curr. Opin. Cell Biol. 10: 339-345.

Gregory, P.D., A. Schmid, M. Zavari, L. Lui, S.L. Berger, and W. Horz. 1998. Absence of Gcn5 HAT activity defines a novel state in the opening of chromatin at the PHO5 promoter in yeast. Mol. Cell 1: 495-505.

Grunstein, M. 1997. Histone acetylation in chromatin structure and transcription. Nature 389: 349-352.

Guyon, J.R., G.J. Narlikar, S. Sif, and R.E. Kingston. 1999. Stable remodeling of tailless nucleosomes by the human SWI-SNF complex. Mol. Cell. Biol. 19: 2088-2097.

Hamiche, A., R. Sandaltzopoulos, D.A. Gdula, and C. Wu. 1999. ATP-dependent histone octamer sliding mediated by the chromatin remodeling complex NURF. Cell 97: 833-842.

Hartzog, G.A. and F. Winston. 1997. Nucleosomes and transcription: recent lessons from genetics. Curr. Opin. Genet. Dev. 7: 192-198.

Hartzog, G.A., T. Wada, H. Handa, and F. Winston. 1998. Evidence that Spt4, Spt5, and Spt6 control transcription elongation by RNA polymerase II in Saccharomyces cerevisiae. Genes \& Dev. 12: 357-369.

Hecht, A., T. Laroche, S. Strahl-Bolsinger, S.M. Gasser, and M. Grunstein. 1995. Histone H3 and H4 N-termini interact with SIR3 and SIR4 proteins: A molecular model for the formation of heterochromatin in yeast. Cell 80: 583-592.

Hirschhorn, J.N., S.A. Brown, C.D. Clark, and F. Winston. 1992. Evidence that SNF2/SWI2 and SNF5 activate transcription in yeast by altering chromatin structure. Genes \& Dev. 6: 2288-2298.

Holstege, F.C., E.G. Jennings, J.J. Wyrick, T.I. Lee, C.J. Hengartner, M.R. Green, T.R., Golub, E.S. Lander, and R.A. Young. 1998. Dissecting the regulatory circuitry of a eukaryotic genome. Cell 95: 717-728.

Ikeda, K., D.J. Steger, A. Eberharter, and J.L. Workman. 1999. Activation domain-specific and general transcription stimulation by native histone acetyltransferase complexes. Mol. Cell. Biol. 19: 855-863.

Imbalzano, A.N., H. Kwon, M.R. Green, and R.E. Kingston. 1994. Facilitated binding of TATA-binding protein to nucleosomal DNA. Nature 370: 481-485.

Imbalzano, A.N., G.R. Schnitzler, and R.E. Kingston. 1996. Nucleosome disruption by human SWI/SNF is maintained in the absence of continued ATP hydrolysis. J. Biol. Chem. 271: 20726-20733.

Imhof, A. and A.P. Wolffe. 1998. Transcription: Gene control by targeted histone acetylation. Curr. Biol. 8: R422-R424.

Ito, T., M. Bulger, M.J. Pazin, R. Kobayashi, and J.T. Kadonaga. 1997. ACF, an ISWI-containing and ATP-utilizing chromatin assembly and remodeling factor. Cell 90: 145-155.

Ito, T., M.E. Levenstein, D.V. Fyodorov, A.K. Kutach, R. Kobayashi, and J.T. Kadonaga. 1999. ACF consists of two subunits, acf1 and ISWI, that function cooperatively in the ATPdependent catalysis of chromatin assembly. Genes \& Dev. 13: 1529-1539. 
Kadonaga, J.T. 1998. Eukaryotic transcription: An interlaced network of transcription factors and chromatin-modifying machines. Cell 92: 307-313.

Kadosh, D. and K. Struhl. 1997. Repression by Ume6 involves recruitment of a complex containing $\operatorname{Sin} 3$ corepressor and Rpd3 histone deacetylase to target promoters. Cell 89: 365371 .

- 1998. Histone deacetylase activity of Rpd3 is important for transcriptional repression in vivo. Genes \& Dev. 12: $797-$ 805.

Kamakaka, R.T., M. Bulger, and J.T. Kadonaga. 1993. Potentiation of RNA polymerase II transcription by Gal4-VP16 during but not after DNA replication and chromatin assembly. Genes \& Dev. 7: 1779-1795.

Kehle, J., D. Beuchle, S. Treuheit, B. Christen, J.A. Kennison, M. Bienz, and J. Muller. 1998. dMi-2, a hunchback-interacting protein that functions in polycomb repression. Science 282: 1897-1900.

Khavari, P.A., C.L. Peterson, J.W. Tamkun, and G.R. Crabtree. 1993. BRG1 contains a conserved domain of the SWI2/SNF2 family necessary for normal mitotic growth and transcription. Nature 366: 170-174.

Kim, J., S. Sif, B. Jones, A. Jackson, J. Koipally, E. Heller, S. Winandy, A. Viel, A. Sawyer, A., T. Ikeda, R. Kingston, and K. Georgopoulos. 1999. Ikaros DNA binding proteins direct formation of chromatin remodeling complexes in lymphocytes. Immunity 10: 345-355.

Kingston, R.E., C.A. Bunker, and A.N. Imbalzano. 1996. Repression and activation by multiprotein complexes that alter chromatin structure. Genes \& Dev. 10: 905-920.

Kraus, W.L. and J.T. Kadonaga. 1998. p300 and estrogen receptor cooperatively activate transcription via differential enhancement of initiation and reinitiation. Genes \& Dev. 12: 331342.

Krebs J.E., M.H. Kuo, C.D. Allis, and C.L. Peterson. 1999. Cell cycle-regulated histone acetylation required for expression of the yeast $H O$ gene. Genes \& Dev. 13: 1412-1421.

Kuo, M.H. and C.D. Allis. 1998. Roles of histone acetyltransferases and deacetylases in gene regulation. BioEssays 20: 615-626.

Kuo, M.H., J. Zhou, P. Jambeck, M.E. Churchill, and C.D. Allis. 1998. Histone acetyltransferase activity of yeast Gen $5 p$ is required for the activation of target genes in vivo. Genes \& Dev. 12: 627-639.

Kwon, H., A.N. Imbalzano, P.A. Khavari, R.E. Kingston, and M.R. Green. 1994. Nucleosome disruption and enhancement of activator binding by a human SWI/SNF complex. Nature 370: 477-481.

Langst, G., E.J. Bonte, D.F. Corona, and P.B. Becker. 1999. Nucleosome movement by CHRAC and ISWI without disruption or trans-displacement of the histone octamer. Cell 97: 843-852.

Laurent, B.C., I. Treich, and M. Carlson. 1993. The yeast SNF2/ SWI2 protein has DNA-stimulated ATPase activity required for transcriptional activation. Genes \& Dev. 7: 583-591.

Lee, D.Y., J.J. Hayes, D. Pruss, and A.P. Wolffe. 1993. A positive role for histone acetylation in transcription factor access to nucleosomal DNA. Cell 72: 73-84.

LeRoy, G., G. Orphanides, W.S. Lane, and D. Reinberg. 1998. Requirement of RSF and FACT for transcription of chromatin templates in vitro. Science 282: 1900-1904.

Logie, C. and C.L. Peterson. 1997. Catalytic activity of the yeast SWI/SNF complex on reconstituted nucleosome arrays. EMBO I. 16: 6772-6782.

Logie, C., C. Tse, J.C. Hansen, and C.L. Peterson. 1999. The core histone $\mathrm{N}$-terminal domains are required for multiple rounds of catalytic chromatin remodeling by the SWI/SNF and RSC complexes. Biochemistry 38: 2514-2522.

Lorch, Y., B.R. Cairns, M. Zhang, and R.D. Kornberg. 1998. Activated RSC-nucleosome complex and persistently altered form of the nucleosome. Cell 94: 29-34.

Lorch, Y., M. Zhang, and R.D. Kornberg. 1999. Histone octamer transfer by a chromatin-remodeling complex. Cell 96: 389392.

Meersseman, G., S. Pennings. and E.M. Bardbury. 1992. Mobile nucleosomes-A general behavior. EMBO J. 11: 2951-2959.

Mizuguchi, G., T. Tsukiyama, J. Wisniewski, and C. Wu. 1997. Role of nucleosome remodeling factor NURF in transcriptional activation of chromatin. Mol. Cell 1: 141-150.

Mizzen, C.A. and C.D. Allis. 1998. Linking histone acetylation to transcriptional regulation. Cell. Mol. Life Sci. 54: 6-20.

Moreira, J.M. and S. Holmberg. 1999. Transcriptional repression of the yeast CHAl gene requires the chromatin-remodeling complex RSC. EMBO J. 18: 2836-2844.

Muchardt, C. and M. Yaniv. 1993. A human homologue of Saccharomyces cerevisiae SNF2/SWI2 and Drosophila brm genes potentiates transcriptional activation by the glucocorticoid receptor. EMBO J. 12: 4279-4290.

Muchardt, C., J.C. Reyes, B. Bourachot, E. Leguoy, and M. Yaniv. 1996. The hbrm and BRG-1 proteins, components of the human SNF/SWI complex, are phosphorylated and excluded from the condensed chromosomes during mitosis. EMBO I. 15: 3394-3402.

Murphy, D.J., S. Hardy, and D.A. Engel. 1999. Human SWI-SNF component BRG1 represses transcription of the c-fos gene. Mol. Cell. Biol. 19: 2724-2733.

Mymryk, J.S. and T.K. Archer. 1995. Dissection of progesterone receptor-mediated chromatin remodeling and transcriptional activation in vivo. Genes \& Dev. 9: 1366-1376.

Naar, A.M., P.A. Beaurang, S. Zhou, S. Abraham, W. Solomon, and R. Tjian. 1999. Composite co-activator ARC mediates chromatin-directed transcriptional activation. Nature 398: 828-832.

Ogryzko, V.V., T. Kotani, X. Zhang, R.L. Schlitz, T. Howard, X.J. Yang, B.H. Howard, J. Qin, and Y. Nakatani. 1998. Histonelike TAFs within the PCAF histone acetylase complex. Cell 94: $35-44$.

Orphanides, G., G. LeRoy, C.H. Chang, D.S. Luse, and D. Reinberg. 1998. FACT, a factor that facilitates transcript elongation through nucleosomes. Cell 92: 105-116.

Ostlund, F.A., P. Blomquist, H. Kwon, and O. Wrange. 1997. Glucocorticoid receptor-glucocorticoid response element binding stimulates nucleosome disruption by the SWI/SNF complex. Mol. Cell. Biol. 17: 895-905.

Owen-Hughes, T. and J.L. Workman. 1996. Remodeling the chromatin structure of a nucleosome array by transcription factor-targeted trans-displacement of histones. EMBO $\mathrm{J}$. 15: 4702-4712.

Owen-Hughes, T., R.T. Utley, J. Cote, C.L. Peterson, and J.L. Workman. 1996. Persistent site-specific remodeling of a nucleosome array by transient action of the SWI/SNF complex. Science 273: 513-516.

Papoulas, O., S.J. Beek, S.L. Moseley, C.M. McCallum, M. Sarte, A. Shearn, and J.W. Tamkun. 1998. The Drosophila trithorax group proteins BRM, ASH1 and ASH2 are subunits of distinct protein complexes. Development 125: 3955-3966.

Peterson, C.L., A. Dingwall, and M.P. Scott. 1994. Five SWI/ SNF gene products are components of a large multiprotein complex required for transcriptional enhancement. Proc. Nat1. Acad. Sci. 91: 2905-2908.

Peterson, C.L., Y. Zhao, and B.T. Chait. 1998. Subunits of the yeast SWI/SNF complex are members of the actin-related 
protein (ARP) family. J. Biol. Chem. 273: 23641-23644.

Phelan, M.L., S. Sif, G.J. Narlikar, and R.E. Kingston. 1999. Reconstitution of a core chromatin remodeling complex from SWI/SNF subunits. Mol. Cell 3: 247-253.

Pina, B., U. Bruggemeier, and M. Beato. 1990. Nucleosome positioning modulates accessibility of regulatory proteins to the mouse mammary tumor virus promoter. Cell 60: 719731.

Pollard, K.J. and C.L. Peterson. 1997. Role for ADA/GCN5 products in antagonizing chromatin-mediated transcriptional repression. Mol. Cell. Biol. 17: 6212-6222.

- 1998. Chromatin remodeling: a marriage between two families? BioEssays 20: 771-780.

Rachez, C., B.D. Lemon, Z. Suldan, V. Bromleigh, M. Gamble, A.M. Naar, H. Erdjument-Bromage, P. Tempst, and L.P. Freedman. 1999. Ligand-dependent transcription activation by nuclear receptors requires the DRIP complex. Nature 398: 824-828.

Renauld, H., O.M. Aparicio, P.D. Zierath, B.L. Billington, S.K. Chhablani, and D.E. Gottschling. 1993. Silent domains are assembled continuously from the telomere and are defined by promoter distance and strength, and by SIR3 dosage. Genes \& Dev. 7: 1133-1145.

Roberts, S.M. and F. Winston. 1997. Essential functional interactions of SAGA, a Saccharomyces cerevisiae complex of $\mathrm{Spt}$, Ada and Gen5 proteins, with the Snf/Swi, and Srb/mediator complexes. Genetics 147: 451-465.

Rundlett, S.E., A.A. Carmen, R. Kobayashi, S. Bavykin, B.M. Turner, and M. Grunstein. 1996. HDA1 and RPD3 are members of distinct yeast histone deacetylase complexes that regulate silencing and transcription. Proc. Natl. Acad. Sci. 93: $14503-14508$.

Rundlett, S.E., A.A. Carmen, N. Suka, B.M. Turner, and M. Grunstein. 1998. Transcriptional repression by UME6 involves deacetylation of lysine 5 of histone H4 by RPD3. Nature 392: 831-835.

Schnitzler, G., S. Sif, and R.E. Kingston. 1998. Human SWI/SNF interconverts a nucleosome between its base state and a stable remodeled state. Cell 94: 17-27.

Sheridan, P.L., T.P. Mayall, E. Verdin, and K.A. Jones. 1997. Histone acetyltransferases regulate HIV-1 enhancer activity in vitro. Genes \& Dev. 11: 3327-3340.

Sif, S., P.T. Stukenberg, M.W. Kirschner, and R.E. Kingston. 1998. Mitotic inactivation of a human SWI/SNF chromatin remodeling complex. Genes \& Dev. 12: 2842-2851.

Steger, D.J., A. Eberharter, S. John, P.A. Grant, and J.L. Workman. 1998. Purified histone acetyltransferase complexes stimulate HIV-1 transcription from preassembled nucleosomal arrays. Proc. Nat1. Acad. Sci. 95: 12924-12929.

Sterner, D.E., P.A. Grant, S.M. Roberts, L.J. Duggan, R. Belotserkovskaya, L.A. Pacella, F. Winston, J.L. Workman, and S.L. Berger. 1999. Functional organization of the yeast SAGA complex: distinct components involved in structural integrity, nucleosome acetylation, and TATA-binding protein interaction. Mol. Cell. Biol. 19: 86-98.

Struhl, K. 1998. Histone acetylation and transcriptional regulatory mechanisms. Genes \& Dev. 12: 599-606.

Sudarsanam, P., Y. Cao, L. Wu, B.C. Laurent, and F. Winston. 1999. The nucleosome remodeling complex, Snf/Swi, is required for the maintenance of transcription in vivo and is partially redundant with the histone acetyltransferase, Gcn5. EMBO I. 18: 3101-3106.

Tamkun, J.W. 1995. The role of brahma and related proteins in transcription and development. Curr. Opin. Genet. Dev. 5: 473-477.

Tong, J.K., C.A. Hassig, G.R. Schnitzler, R.E. Kingston, and S.L.
Schreiber. 1998. Chromatin deacetylation by an ATP-dependent nucleosome remodelling complex. Nature 395: 917921.

Tsukiyama, T. and C. Wu. 1995. Purification and properties of an ATP-dependent nucleosome remodeling factor. Cell 83: 1011-1020.

-1997. Chromatin remodeling and transcription. Curr. Opin. Genet. Dev. 7: 182-191.

Tsukiyama, T., P.B. Becker, and C. Wu. 1994. ATP-dependent nucleosome disruption at a heat-shock promoter mediated by binding of GAGA transcription factor. Nature 367: 525532.

Tsukiyama, T., C. Daniel, J. Tamkun, and C. Wu. 1995. ISWI, a member of the SWI2/SNF2 ATPase family, encodes the 140 $\mathrm{kDa}$ subunit of the nucleosome remodeling factor. Cell 83: 1021-1026.

Tsukiyama, T., J. Palmer, C.C. Landel, J. Shiloach, and C. Wu. 1999. Characterization of the imitation switch subfamily of ATP-dependent chromatin-remodeling factors in Saccharomyces cerevisiae. Genes \& Dev. 13: 686-697.

Utley, R.T., K. Ikeda, P.A. Grant, J. Cote, D.J. Steger, A. Eberharter, S. John, and J.L. Workman. 1998. Transcriptional activators direct histone acetyltransferase complexes to nucleosomes. Nature 394: 498-502.

Varga-Weisz, P.D. and P.B. Becker. 1998. Chromatin-remodeling factors: Machines that regulate? Curr. Opin. Cell Biol. 10: 346-353.

Varga-Weisz, P.D., T.A. Blank, and P.B. Becker. 1995. Energydependent chromatin accessibility and nucleosome mobility in a cell-free system. EMBO J. 14: 2209-2216.

Varga-Weisz, P.D., M. Wilm, E. Bonte, K. Dumas, M. Mann, and P.B. Becker. 1997. Chromatin-remodelling factor CHRAC contains the ATPases ISWI and topoisomerase II. Nature 388: 598-602.

Vettese-Dadey, M., P. Walter, H. Chen, L.-J. Juan, and J.L. Workman. 1994. Role of the histone amino termini in facilitated binding of a transcription factor, GAL4-AH, to nucleosome cores. Mol. Cell. Biol. 14: 970-981.

Wada, T., T. Takagi, Y. Yamaguchi, A. Ferdous, T. Imai, S. Hirose, S. Sugimoto, K. Yano, G.A. Hartzog, F. Winston, S. Buratowski, and H. Handa. 1998. DSIF, a novel transcription elongation factor that regulates RNA polymerase II processivity, is composed of human Spt4 and Spt5 homologs. Genes \& Dev. 12: 343-356.

Wade, P.A., P.L. Jones, D. Vermaak, and A.P. Wolffe. 1998. A multiple subunit Mi-2 histone deacetylase from Xenopus laevis cofractionates with an associated Snf2 superfamily ATPase. Curr. Biol. 8: 843-846.

Wang, W., J. Cote, Y. Xue, S. Zhou, P.A. Khavari, S.R. Biggar, C. Muchardt, G.V. Kalpana, S.P. Goff, M. Yaniv, J.L. Workman, and G.R. Crabtree. 1996a. Purification and biochemical heterogeneity of the mammalian SWI-SNF complex. EMBO $\mathrm{I}$. 15: 5370-5382.

Wang, W., Y. Xue, S. Zhou, A. Kuo, B.R. Cairns, and G.R. Crabtree. 1996b. Diversity and specialization of mammalian SWI/SNF complexes. Genes \& Dev. 10: 2117-2130.

Wilson, C.J., D.M. Chao, A.N. Imbalzano, G.R., Schnitzler, R.E. Kingston, and R.A.Young. 1996. RNA polymerase II holoenzyme contains SWI/SNF regulators involved in chromatin remodeling. Cell 84: 235-244.

Winston, F. and M. Carlson. 1992. Yeast SNF/SWI transcriptional activators and the SPT/SIN chromatin connection. Trends Genet. 8: 387-391.

Wong, J., Y.B. Shi, and A.P. Wolffe. 1997. Determinants of chromatin disruption and transcriptional regulation instigated by the thyroid hormone receptor: Hormone-regulated chro- 
Kingston and Narlikar

matin disruption is not sufficient for transcriptional activation. EMBO J. 16: 3158-3171.

Workman, J.L. and R.E. Kingston. 1998. Alteration of nucleosome structure as a mechanism of transcriptional regulation. Annu. Rev. Biochem. 67: 545-579.

Workman, J.L., I.C.A. Taylor, and R.E. Kingston. 1991. Activation domains of stably bound GAL4 derivatives alleviate repression of promoters by nucleosomes. Cell 64: 533-544.

Xue, Y., J. Wong, G.T. Moreno, M.K. Young, J. Cote, and W. Wang. 1998. NURD, a novel complex with both ATP-dependent chromatin-remodeling and histone deacetylase activities. Mol. Cell 2: 851-861.

Yoshinaga, S.K., C.L. Peterson, I. Herskowitz, and K.R. Yamamoto. 1992. Roles of SWI1, SWI2, and SWI3 proteins for transcriptional enhancement by steroid receptors. Science 258: 1598-1604.

Zhang, W., J.R. Bone, D.G. Edmondson, B.M. Turner, and S.Y. Roth. 1998a. Essential and redundant functions of histone acetylation revealed by mutation of target lysines and loss of the Gcn5p acetyltransferase. EMBO J. 17: 3155-3167.

Zhang, Y., G. LeRoy, H.P. Seelig, W.S. Lane, and D. Reinberg. 1998b. The dermatomyositis-specific autoantigen Mi2 is a component of a complex containing histone deacetylase and nucleosome remodeling activities. Cell 95: 279-289.

Zhao, K., W. Wang, O.J. Rando, Y. Xue, K. Swiderek, A. Kuo, and G.R. Crabtree. 1998. Rapid and phosphoinositol-dependent binding of the SWI/SNF-like BAF complex to chromatin after T lymphocyte receptor signaling. Cell 95: 625-636. 


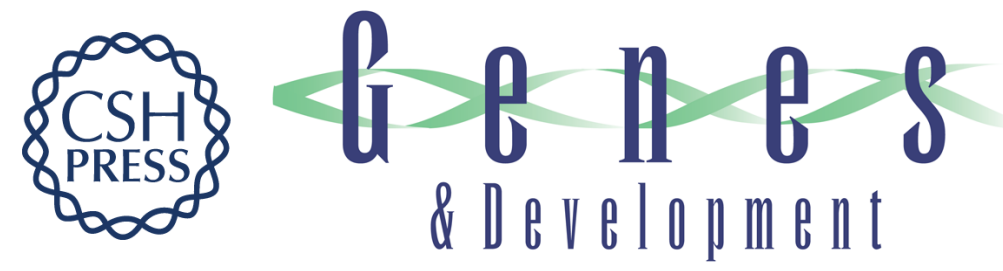

\section{ATP-dependent remodeling and acetylation as regulators of chromatin fluidity}

Robert E. Kingston and Geeta J. Narlikar

Genes Dev. 1999, 13:

References This article cites 122 articles, 57 of which can be accessed free at:

http://genesdev.cshlp.org/content/13/18/2339.full.html\#ref-list-1

License

Email Alerting Receive free email alerts when new articles cite this article - sign up in the box at the top Service right corner of the article or click here.

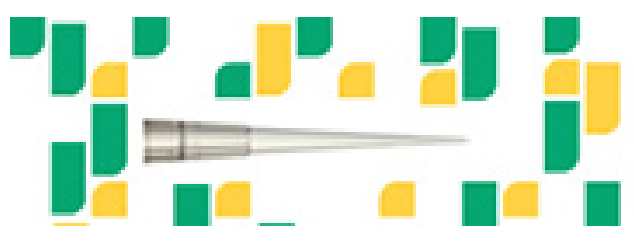

Focused on your science. 